\title{
Case Report: Spinal Subarachnoid Hemorrhage: A Rare Complication of Hemorrhagic Fever with Renal Syndrome
}

\author{
Shih-Hao Lo, ${ }^{1}$ Pei-Ting Chen, ${ }^{2}$ Wan-Jin Yu, ${ }^{3}$ Ke-Syuan Hsieh, ${ }^{3}$ and Tun-Chieh Chen ${ }^{2,3,4 *}$ \\ ${ }^{1}$ Division of Infectious Diseases, Department of Internal Medicine, Kaohsiung Medical University Hospital, Kaohsiung Medical University, \\ Kaohsiung, Taiwan; ${ }^{2}$ Department of Post Baccalaureate Medicine, College of Medicine, Kaohsiung Medical University, Kaohsiung, Taiwan; \\ ${ }^{3}$ Department of Medicine, College of Medicine, Kaohsiung Medical University, Kaohsiung, Taiwan; ${ }^{4}$ Department of Internal Medicine, Kaohsiung \\ Municipal Ta-Tung Hospital, Kaohsiung Medical University, Kaohsiung, Taiwan
}

\begin{abstract}
Hemorrhagic fever with renal syndrome (HFRS), caused by hantavirus, is occasionally seen in tropical areas. The virus is carried by specific rodent host species. Hemorrhagic fever with renal syndrome is characterized by renal failure and hemorrhagic manifestations, and its complications may be severe, including massive bleeding, multi-organ dysfunction, and possibly death. In this patient case, a 46-year-old woman diagnosed with HFRS initially presented with fever, impaired renal function, and thrombocytopenia. Four days after symptom onset, the patient complained of abrupt right lower abdominal pain and numbness. Magnetic resonance imaging revealed a spinal subarachnoid hemorrhage (SAH) beyond the T7 to S2 vertebrae. No cases of spinal SAH in HFRS have been reported until now. This case demonstrates that when a patient's symptoms are atypical, bleeding-related complications must be considered.
\end{abstract}

\section{INTRODUCTION}

Hantavirus, which causes hemorrhagic fever with renal syndrome (HFRS) and hantavirus cardiopulmonary syndrome, is carried by specific rodent host species. The virus infects humans via inhalation of aerosols or dust particles contaminated with virus-laden rodent excretions. ${ }^{1}$ Hemorrhagic fever with renal syndrome is characterized by renal failure and hemorrhagic manifestations, and its clinical presentation ranges from subclinical to severe disease. Here, we report a case of HFRS with a rare complication of spinal subarachnoid hemorrhage (SAH).

\section{CASE REPORT}

A 46-year-old woman who sold tofu in a traditional market and reported being generally healthy presented with intermittent fever, headache, and lower abdominal pain 3 days before admission (symptom onset as day 1, Figure 1A). She denied recent travel history or contact with soil, bodies of water, animals, or chemicals. However, she reported cleaning up rodent feces in the market without using protection. Her vital signs at our emergency department were as follows: body temperature $39.3^{\circ} \mathrm{C}$, blood pressure 163/106 $\mathrm{mmHg}$, pulse 119/minute, and respiratory rate $16 /$ minute. Physical examination revealed abdominal tenderness at the right upper quadrant and right costovertebral angle, knocking pain. The initial laboratory assessment showed a normal blood cell count, except thrombocytopenia with a platelet count of $136,000 / \mu \mathrm{L}$, and normal liver and renal function test results. The results of prothrombin time and partial thromboplastin time were in a normal range. Urinalysis showed microhematuria but no pyuria. The chest $\mathrm{X}$-ray also appeared normal. Computed tomography of the abdomen disclosed bilateral hydronephrosis, mild infiltration in the pelvic region with pelvic ascites, and a suspicious right corpus luteal cyst. One week

* Address correspondence to Tun-Chieh Chen, Department of Internal Medicine, Kaohsiung Municipal Ta-Tung Hospital, Kaohsiung Medical University, Taiwan No. 68, Jhonghua 3rd Rd., Cianjin District, Kaohsiung City 80145, Taiwan. E-mail: kmtthidchen@gmail.com later, an intermittent fever remained with progressive thrombocytopenia with a minimum platelet count of 25000/ $\mu \mathrm{L}$, deteriorated renal function with increased creatinine to $1.98 \mathrm{mg} / \mathrm{dL}$, and elevated liver enzymes including aspartate aminotransferase $(425 \mathrm{lU} / \mathrm{mL})$ and alanine aminotransferase $(215 \mathrm{IU} / \mathrm{mL})$. There was no gross hematuria or tarry stool, but evidence of easy bruising was found over the limbs. Ceftriaxone and doxycycline were prescribed under the suspicion of leptospirosis, rickettsia disease, and hantavirus infection-conditions that were also reported to the Taiwan CDC. The serology report from the Taiwan CDC showed the equivocal result of hantavirus IgM and negative for IgG, which is screened for using pan-hantavirus IgG and IgM DxSelect ELISAs (Focus Technologies, Cypress Hill, CA) that have an overall sensitivity and specificity more than $94 \%$. $^{2}$ The results of other disease screens were negative. The platelet count, renal function, and liver enzyme levels gradually recovered. However, she complained of suddenonset severe right lower abdominal pain extending to the right inguinal area, external thigh, and lower leg on day 10. Urine retention and decreased muscle power of the right lower proximal limb were noted despite antibiotic treatment. The diagnosis of HFRS was confirmed after the secondary report of serum hantavirus IgM and IgG turned to be positive 10 days after the first report; however, this finding could not explain the aforementioned symptoms. Magnetic resonance imaging (MRI) of the thoracolumbar spine for a suspected spinal cord lesion on day 19 revealed a late subacute $\mathrm{SAH}$ beyond the T7 to $\mathrm{S} 2$ level and bulging of the L3-4, L4-5, and L5-S1 discs, causing mild thecal sac compression (Figure 1). One week after her abdominal pain resolved, her thrombocytopenia $(383000 / \mu \mathrm{L})$, renal function (creatinine, $0.43 \mathrm{mg} / \mathrm{dL}$ ), and the liver enzymes (AST/ALT, $37 / 35 \mathrm{IU} / \mathrm{L}$ ) each recovered to normal. A follow-up MRI performed 6 weeks later showed interval remission of the $\mathrm{SAH}$ in the thoracolumbar spine. Her pain and weakness also resolved.

\section{DISCUSSION}

Hemorrhagic fever with renal syndrome was not reported in Taiwan before its first case in $1995 .{ }^{3}$ According to the Taiwan 

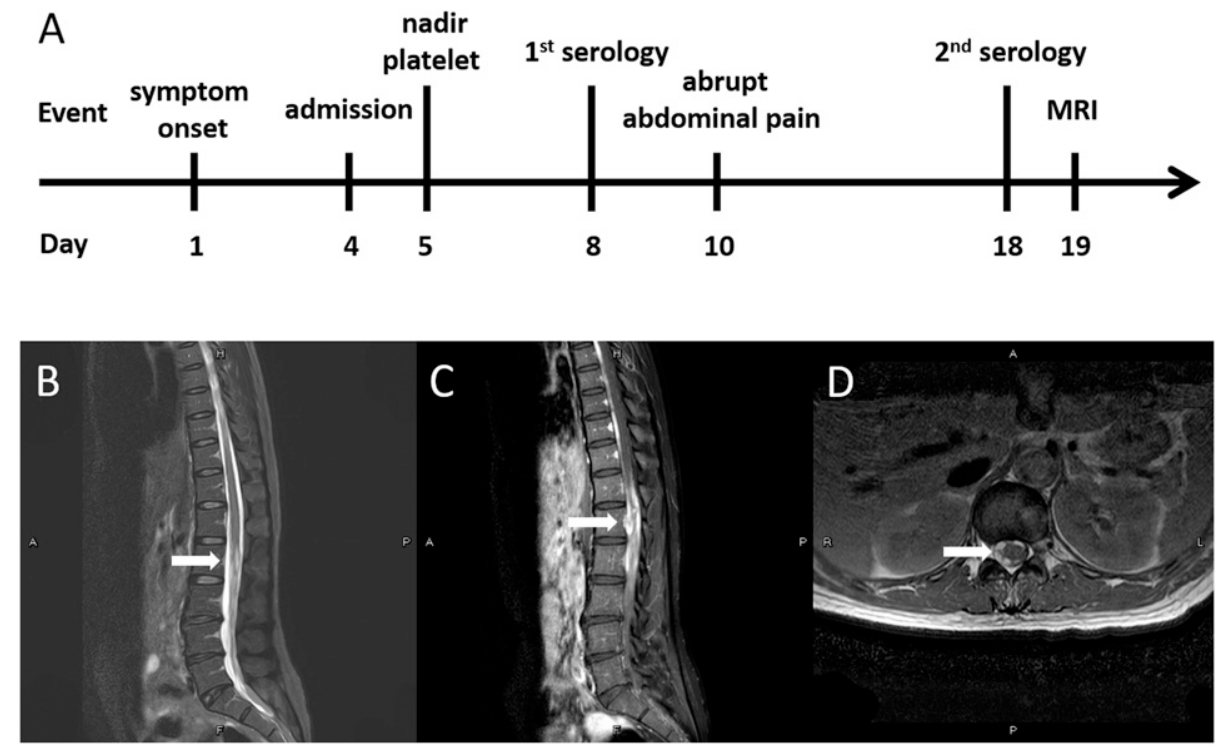

FIGURE 1. Event time schedule (A) and the magnetic resonance imaging (MRI) of the thoracolumbar spine on day 19 revealed mild hyperintense on T2WI (B) and heterogeneous hyperintense on T1WI from T7 to S2 (C and D), considering the late subacute stage of a subarachnoid hemorrhage.

CDC report, since 2001 , there have only been sporadic cases each year. Most cases were local and more common in males. ${ }^{4}$ The diseases identified in Taiwan were most closely related to the prototype Seoul strain virus (SEOV); no cases of Hantaan strain virus were discovered from any rodent species indigenous to Taiwan. ${ }^{5}$ A typical course of HFRS can be divided into five distinct phases: febrile, hypotensive, oliguric, polyuric, and convalescent. Infection with SEOV causes a moderate form of HFRS that makes it difficult to distinguish among the five phases, is often associated with the presence of hepatitis, and has a mortality rate of $<1 \%$. $^{1,6,7}$

Hemorrhagic fever with renal syndrome may induce vascular endothelial dysfunction, which is characterized by a dramatic increase in vascular permeability. Increased vascular permeability leads to plasma exosmosis and even hemorrhage, which is associated with many clinical features in HFRS. Abdominal pain with ascites, as in our case, may be explained by the aforementioned phenomenon. Hemorrhagic fever with renal syndrome also features various complications including acute encephalomyelitis, bleeding, multi-organ dysfunction, pituitary hemorrhage, glomerulonephritis, pulmonary edema, shock, acute respiratory distress syndrome, disseminated intravascular coagulation, and death. ${ }^{8,9}$ A review of 811 cases of HFRS caused by Puumala virus revealed that nine patients (1\%) had severe neurological manifestations, with meningism and cerebral hemorrhage occurring during the first week of illness, whereas epileptiform seizures and urinary bladder paralysis developed during the second week. There was only one fatality due to cerebral hemorrhage. ${ }^{10}$ To date, only one case of intracranial SAH caused by HFRS has been reported. ${ }^{11}$

Spinal SAH is rare and constitutes only about $0.05-1.5 \%$ of all case of $\mathrm{SAH} .{ }^{12} \mathrm{Kreppel} \mathrm{et} \mathrm{al.}^{13}$ determined that spinal SAH was more commonly seen in young patients with etiologies including tumor-related bleeding, vascular formation, coagulopathy, and puncture-related bleeding. In a literature review of a total of 69 cases of spinal SAH, $40.5 \%$ had coagulopathies that were either pharmacologically induced or resulting from systemic diseases. ${ }^{14}$ Sudden back pain or headache, acute sciatic pain, paraparesis, sensory disturbances, and sphincter disturbances are described as clinical presentations in most published reports on spinal SAH. ${ }^{15}$ In the case presented herein, right flank pain with extension to the inguinal area and paresthesia over the right thigh were the initial manifestations of spinal $\mathrm{SAH}$, which started about 9 days after fever onset. Later, the thoracolumbar MRI showed T7-S2 spinal SAH with hyperintensity on both T1 and T2 sequencing, which indicated a late subacute hemorrhagic event compatible with the clinical course.

The etiologies of spinal SAH include neoplasms, vascular lesions, trauma, coagulopathy, and systemic diseases such as infection, vasculitis, and connective tissue disorders. ${ }^{16}$ No reports have described spinal SAH caused by hantavirus infection. The possible mechanism behind this is the thrombocytopenia, which reportedly occurred in $83 \%$ of patients with SEOV infection. ${ }^{7}$ Besides, impaired platelet functions of aggregation and release, disturbance of blood coagulation, production of immune complexes, and cytokines that damage the small blood vessels are also contributors. ${ }^{11,17}$ Here, we reported a case of spinal SAH caused by HFRS with unexpected initial presentations.

Received July 9, 2020. Accepted for publication January 4, 2021.

Published online February 16, 2021.

Acknowledgments: We would like to thank the staff from the Department of Medical Imaging, Kaohsiung Medical University Hospital, and Kaohsiung Medical University for their assistance in this study.

Authors' addresses: Shih-Hao Lo, Division of Infectious Diseases, Department of Internal Medicine, Kaohsiung Medical University Hospital, Kaohsiung Medical University, Kaohsiung City, Taiwan, E-mail: pipy0529@gmail.com. Pei-Ting Chen, Department of Post Baccalaureate Medicine, College of Medicine, Kaohsiung Medical University, Kaohsiung, Taiwan, E-mail: b96207045@gmail.com. WanJin Yu and Ke-Syuan Hsieh, Department of Medicine, College of Medicine, Kaohsiung Medical University, Kaohsiung, Taiwan, E-mails: winniestatham@gmail.com and gg31540@gmail.com. Tun-Chieh Chen, Department of Internal Medicine, Kaohsiung Municipal TaTung Hospital, Kaohsiung Medical University, Taiwan, E-mail: kmtthidchen@gmail.com. 


\section{REFERENCES}

1. Avsic-Zupanc T, Saksida A, Korva M, 2019. Hantavirus infections. Clin Microbiol Infect 21S: e6-e16.

2. Goeijenbier M et al., 2014. The hanta hunting study: underdiagnosis of Puumala hantavirus infections in symptomatic non-travelling leptospirosis-suspected patients in The Netherlands, in 2010 and April to November 2011. Euro Surveill 19: 20878.

3. Wu TN, Chin C, Shen CY, Chang PY, 1996. Hantavirus infection in Taiwan. Lancet 347: 770-771.

4. Taiwan National Infectious Disease Statistics System. Available at: https://nidss.cdc.gov.tw/en/SingleDisease.aspx?dc $=1 \& \mathrm{dt}=2 \&$ disease $=0786$. Accessed December 11, 2020.

5. Chin C et al., 2000. Hantavirus infection in Taiwan: the experience of a geographically unique area. J Med Virol 60: 237-247.

6. Park SC et al., 1989. A clinical study of hemorrhagic fever with renal syndrome caused by Seoul virus infection. Korean J Intern Med 4: 130-135.

7. Jonsson CB, Figueiredo LT, Vapalahti O, 2010. A global perspective on hantavirus ecology, epidemiology, and disease. Clin Microbiol Rev 23: 412-441.

8. Jiang $\mathrm{H}$, Zheng $X$, Wang L, Du H, Wang P, Bai X, 2017. Hantavirus infection: a global zoonotic challenge. Virol $\operatorname{Sin} 32$ : 32-43.

9. Jiang H, Du H, Wang LM, Wang PZ, Bai XF, 2016. Hemorrhagic fever with renal syndrome: pathogenesis and clinical picture. Front Cell Infect Microbiol 6: 1.
10. Alexeyev OA, Morozov VG, 1995. Neurological manifestations of hemorrhagic fever with renal syndrome caused by Puumala virus: review of 811 cases. Clin Infect Dis 20: 255-258.

11. Xu Z, Xu P, Lei X, Xu Z, Wu Q, Zhang J, 2011. Subarachnoid hemorrhage associated with epidemic hemorrhagic fever: a rare case report. Int J Med Sci 8: 640-642.

12. Komiyama M, Yasui T, Sumimoto T, Fu Y, 1997. Spontaneous spinal subarachnoid hematoma of unknown pathogenesis: case reports. Neurosurgery 41: 691-693; discussion 3-4.

13. Kreppel D, Antoniadis G, Seeling W, 2003. Spinal hematoma: a literature survey with meta-analysis of 613 patients. Neurosurg Rev 26: 1-49.

14. Domenicucci M, Ramieri A, Paolini S, Russo N, Occhiogrosso G, Di Biasi C, Delfini R, 2005. Spinal subarachnoid hematomas: our experience and literature review. Acta Neurochir (Wien) 147: 741-750; discussion 50.

15. Kim YH, Cho KT, Chung CK, Kim HJ, 2004. Idiopathic spontaneous spinal subarachnoid hemorrhage. Spinal Cord 42: 545-547.

16. Maiti TK, Bir SC, Nanda A, 2017. Spinal subarachnoid hemorrhage and aneurysms. Handb Clin Neurol 143: 215-223.

17. Xiang LB, Zhang ZY, Chen JM, Xiang JM, Cosgriff TM, 1990. Epidemic hemorrhagic fever. The mechanism of coagulation and fibrinolysis. Chin Med J (Engl) 103: 391-395. 